\title{
Multiplicity of Solutions to a Potential Operator Equation and Its Applications
}

\author{
Jincheng Huang ${ }^{1,2}$ \\ ${ }^{1}$ College of Sciences, Hohai University, Nanjing 210098, China \\ ${ }^{2}$ Department of Mathematics and Physics, Hohai University, Changzhou Campus, Changzhou 213022, China
}

Correspondence should be addressed to Jincheng Huang; slbhuangjc@163.com

Received 12 July 2014; Accepted 27 August 2014; Published 22 October 2014

Academic Editor: M. Victoria Otero-Espinar

Copyright (c) 2014 Jincheng Huang. This is an open access article distributed under the Creative Commons Attribution License, which permits unrestricted use, distribution, and reproduction in any medium, provided the original work is properly cited.

We consider the multiplicity of solutions for operator equation involving homogeneous potential operators. With the help of Nehari manifold and fibering maps, we prove that such equation has at least two nontrivial solutions. Furthermore, we apply this result to prove the existence of two nonnegative solutions for three types of quasilinear elliptic systems involving $(p, q)$-Laplacian operator and concave-convex nonlinearities.

\section{Introduction}

Let $\Omega \subset \mathbb{R}^{N}$ be a bounded domain and $1<t<+\infty$. We define the Sobolev space $W^{1, t}(\Omega)$ equipped with the norm

$$
\|u\|_{t}=\left(\int_{\Omega}\left(|\nabla u|^{t}+|u|^{t}\right) d x\right)^{1 / t}
$$

Then we denote $X=W^{1, p}(\Omega) \times W^{1, q}(\Omega)$ and, for $(u, v) \in X$,

$$
\|(u, v)\|_{X}=\|u\|_{p}+\|v\|_{q} .
$$

It is well known that $X$ is a reflexive Banach space. The dual space of $X$ is denoted by $X^{*}$.

In this paper, we consider the multiplicity results for nonzero solutions of the operator equation

$$
A(u, v)-B(u, v)-C(u, v)=0, \quad(u, v) \in X,
$$

where $A: X \rightarrow X^{*}$ is a $(p, q)$-Laplacian operator defined by $\langle A(u, v),(u, v)\rangle=\|u\|_{p}^{p}+\|v\|_{q}^{q}$ and $B, C: X \rightarrow X^{*}$ are homogeneous operators of degrees $r-1$ and $s-1$. It is well known that the functionals corresponding to $A, B$, and $C$ are given by

$$
\begin{gathered}
a(u, v)=\frac{1}{p}\|u\|_{p}^{p}+\frac{1}{q}\|v\|_{q}^{q}, \\
b(u, v)=\frac{1}{r}\langle B(u, v),(u, v)\rangle, \\
c(u, v)=\frac{1}{s}\langle C(u, v),(u, v)\rangle .
\end{gathered}
$$

Throughout the paper, we will assume the following.

$\left(\mathrm{H}_{1}\right) 1<s<p \leq q<r$.

$\left(\mathrm{H}_{2}\right)$ There exist $\left(u_{i}, v_{i}\right) \in X(i=1,2)$ such that

$$
\left\langle B\left(u_{1}, v_{1}\right),\left(u_{1}, v_{1}\right)\right\rangle>0, \quad\left\langle C\left(u_{2}, v_{2}\right),\left(u_{2}, v_{2}\right)\right\rangle>0 .
$$

$\left(\mathrm{H}_{3}\right) B, C: X \rightarrow X^{*}$ are strongly continuous.

$\left(\mathrm{H}_{4}\right)$ There exist positive numbers $d_{1}$ and $d_{2}$ such that

$$
\begin{aligned}
& \langle B(u, v),(u, v)\rangle \leq d_{1}\left(\|u\|_{p}^{r}+\|v\|_{q}^{r}\right), \\
& \langle C(u, v),(u, v)\rangle \leq d_{2}\left(\|u\|_{p}^{s}+\|v\|_{q}^{s}\right) .
\end{aligned}
$$


There are several studies for existence and multiplicity of solutions of the quasilinear elliptic system

$$
\begin{aligned}
& -\Delta_{p} u=f(u, v), \quad \text { in } \Omega, \\
& -\Delta_{q} v=g(u, v), \quad \text { in } \Omega,
\end{aligned}
$$

where $\Omega$ is a smooth bounded domain of $\mathbb{R}^{N}$. See, for example, [1-7].

In [5], Brown and $\mathrm{Wu}$ considered the semilinear elliptic system

$$
\begin{gathered}
-\Delta u+u=\frac{\alpha}{\alpha+\beta} f(x)|u|^{\alpha-2} u|v|^{\beta}, \quad \text { in } \Omega, \\
-\Delta v+v=\frac{\beta}{\alpha+\beta} f(x)|u|^{\alpha}|v|^{\beta-2} v, \quad \text { in } \Omega, \\
\frac{\partial u}{\partial n}=\lambda g(x)|u|^{q-2} u, \quad \frac{\partial v}{\partial n}=\mu h(x)|v|^{q-2} v, \quad \text { on } \partial \Omega,
\end{gathered}
$$

where $\Omega$ is a smooth bounded domain of $\mathbb{R}^{N}, \alpha, \beta>$ 1 , and $1<q<2<\alpha+\beta<2^{*}$. They found that the above problem has at least two positive solutions if the pair $(\lambda, \mu)$ is below a certain subset of $\mathbb{R}^{2}$.

Recently, Afrouzi and Rasouli [3] considered the semilinear $p$-Laplacian system

$$
\begin{aligned}
& -\Delta_{p} u+m(x)|u|^{p-2} u \\
& =\lambda|u|^{\gamma-2} u+\frac{\alpha}{\alpha+\beta} c(x)|u|^{\alpha-2} u|v|^{\beta}, \quad \text { in } \Omega, \\
& -\Delta_{p} v+m(x)|v|^{p-2} v \\
& =\mu|v|^{\gamma-2} v+\frac{\beta}{\alpha+\beta} c(x)|u|^{\alpha}|v|^{\beta-2} v, \quad \text { in } \Omega, \\
& u=0=v, \quad \text { on } \partial \Omega,
\end{aligned}
$$

where $\alpha, \beta>1,1<\alpha+\beta<p<\gamma<p^{*}$. They proved that the above problem has at least two solutions if $0<|\lambda|^{p /(p-\gamma)}+$ $|\mu|^{p /(p-\gamma)}<\delta^{*}$. Similar considerations can be found in $[1,4$, 6].

We note that few researchers had used variational method with the help of Nehari manifold to consider semilinear elliptic system involving $(p, q)$-Laplacian operator to the best of our knowledge. In this paper, we will use variational method with the help of Nehari manifold and fibering maps (see [8]) to prove the existence of at least two nonzero solutions of problem (3) and then apply this result to three types of $(p, q)$ Laplacian systems. These applications extended some of the results in [3-6].

This paper is organized as follows. In Section 2, we discuss some of the properties of the Nehari manifold for (3). In Section 3, we prove that (3) has at least two nontrivial solutions. Applications to $(p, q)$-Laplacian systems with nonlinearities involving both concave and convex terms are given in the last section.

\section{The Properties of the Nehari Manifold}

We say $(u, v) \in X$ is a weak solution of problem (3) if

$$
\langle A(u, v)-B(u, v)-C(u, v),(w, z)\rangle=0
$$

holds for all $(w, z) \in X$.

Problem (3) has a variational structure. Let $J: X \rightarrow \mathbb{R}$ be defined by

$$
J(u, v)=a(u, v)-b(u, v)-c(u, v),
$$

where $a(u, v), b(u, v)$, and $c(u, v)$ are defined by (4). Clearly, the critical points of $J$ are the weak solutions of problem (3).

As the energy functional $J$ is not bounded below on $X$, it is useful to consider the functional on the Nehari manifold:

$$
\mathscr{N}=\left\{(u, v) \in X \backslash\{(0,0)\}:\left\langle J^{\prime}(u, v),(u, v)\right\rangle=0\right\}
$$

Thus $(u, v) \in \mathcal{N}$ if and only if

$$
\langle A(u, v)-B(u, v)-C(u, v),(u, v)\rangle=0 .
$$

Moreover, we have the following result.

Lemma 1. The energy functional $J$ is coercive and bounded below on $\mathcal{N}$.

Proof. If $(u, v) \in \mathcal{N}$, then

$$
\begin{aligned}
J(u, v)= & a(u, v)-\frac{1}{r}(\langle A(u, v),(u, v)\rangle \\
& \quad-\langle C(u, v),(u, v)\rangle)-c(u, v) \\
= & \left(\frac{1}{p}-\frac{1}{r}\right)\|u\|_{p}^{p}+\left(\frac{1}{q}-\frac{1}{r}\right)\|v\|_{q}^{q} \\
& -\left(\frac{1}{s}-\frac{1}{r}\right)\langle C(u, v),(u, v)\rangle .
\end{aligned}
$$

Thus, by (6),

$$
\begin{gathered}
J(u, v) \geq\left(\frac{1}{p}-\frac{1}{r}\right)\|u\|_{p}^{p}+\left(\frac{1}{q}-\frac{1}{r}\right)\|v\|_{q}^{q} \\
-\left(\frac{1}{s}-\frac{1}{r}\right) d_{2}\|(u, v)\|_{X}^{s} .
\end{gathered}
$$

Without loss of generality, we suppose $\|u\|_{p}>1,\|v\|_{q}>1$; then

$$
J(u, v) \geq C_{1}\|(u, v)\|_{X}^{p}-C_{2}\|(u, v)\|_{X}^{s},
$$

where $C_{1}$ and $C_{2}$ are constants independent of $(u, v)$. Thus $J$ is coercive and bounded below on $\mathcal{N}$.

Define

$$
\begin{aligned}
\Phi(u, v) & =\left\langle J^{\prime}(u, v),(u, v)\right\rangle \\
& =\langle A(u, v)-B(u, v)-C(u, v),(u, v)\rangle .
\end{aligned}
$$


Then, for $(u, v) \in \mathcal{N}$, we have

$$
\begin{aligned}
& \left\langle\Phi^{\prime}(u, v),(u, v)\right\rangle \\
& \quad=(p-s)\|u\|_{p}^{p}+(q-s)\|v\|_{q}^{q}-(r-s)\langle B(u, v),(u, v)\rangle \\
& \quad=(p-r)\|u\|_{p}^{p}+(q-r)\|v\|_{q}^{q}-(s-r)\langle C(u, v),(u, v)\rangle .
\end{aligned}
$$

Now, we split $\mathcal{N}$ into three parts $\mathcal{N}^{+}, \mathcal{N}^{-}$, and $\mathscr{N}^{0}$ defined by

$$
\begin{aligned}
& \mathcal{N}^{+}=\left\{(u, v) \in \mathcal{N} \mid\left\langle\Phi^{\prime}(u, v),(u, v)\right\rangle>0\right\}, \\
& \mathcal{N}^{-}=\left\{(u, v) \in \mathcal{N} \mid\left\langle\Phi^{\prime}(u, v),(u, v)\right\rangle<0\right\}, \\
& \mathcal{N}^{0}=\left\{(u, v) \in \mathcal{N} \mid\left\langle\Phi^{\prime}(u, v),(u, v)\right\rangle=0\right\} .
\end{aligned}
$$
$\mathscr{N}^{0}$.

We now derive some basic properties of $\mathcal{N}^{+}, \mathcal{N}^{-}$, and

Lemma 2. Suppose that $\left(u_{0}, v_{0}\right)$ is a local minimizer for $J$ on $\mathcal{N}$ and $\left(u_{0}, v_{0}\right) \notin \mathscr{N}^{0}$. Then $J^{\prime}\left(u_{0}, v_{0}\right)=0$ in $X^{*}$.

Proof. Consider the optimization problem

$$
\min _{(u, v) \in \mathscr{N}} J(u, v), \quad \text { subject to } \Phi(u, v)=0 .
$$

By the theory of Lagrange multiplier principle, there exists $\mu \in \mathbb{R}$ such that

$$
J^{\prime}\left(u_{0}, v_{0}\right)=\mu \Phi^{\prime}\left(u_{0}, v_{0}\right)
$$

Thus

$$
\left\langle J^{\prime}\left(u_{0}, v_{0}\right),\left(u_{0}, v_{0}\right)\right\rangle=\mu\left\langle\Phi^{\prime}\left(u_{0}, v_{0}\right),\left(u_{0}, v_{0}\right)\right\rangle .
$$

Since $\left(u_{0}, v_{0}\right) \in \mathcal{N}$, we obtain $\left\langle J^{\prime}\left(u_{0}, v_{0}\right),\left(u_{0}, v_{0}\right)\right\rangle=0$. However, the fact $\left(u_{0}, v_{0}\right) \notin \mathcal{N}^{0}$ implies that $\left\langle\Phi^{\prime}\left(u_{0}, v_{0}\right)\right.$, $\left.\left(u_{0}, v_{0}\right)\right\rangle \neq 0$. Thus $\mu=0$ and so $J^{\prime}\left(u_{0}, v_{0}\right)=0$. This completes the proof.

Lemma 3. One has $\mathcal{N}^{0}=\emptyset$ provided

$$
\begin{gathered}
d_{1}^{p-s} d_{2}^{r-p}<(p-s)^{p-s}(r-p)^{r-p}(r-s)^{s-r}, \\
d_{1}^{q-s} d_{2}^{r-q}<(q-s)^{q-s}(r-q)^{r-q}(r-s)^{s-r}, \\
(r-s) d_{2} v_{0}^{s}-(r-q) v_{0}^{q}<-\frac{(p-s)(r-p)}{s} t_{0}^{p}, \\
(r-p) u_{0}^{p}-(r-s) d_{2} u_{0}^{s}>\frac{(q-s)(r-s) d_{2}}{q} \tau_{0}^{s},
\end{gathered}
$$

where

$$
\begin{array}{ll}
v_{0}=\left(\frac{q-s}{(r-s) d_{1}}\right)^{1 /(r-q)}, & t_{0}=\left(\frac{(r-s) s d_{2}}{(r-p) p}\right)^{1 /(p-s)}, \\
u_{0}=\left(\frac{p-s}{(r-s) d_{1}}\right)^{1 /(r-p)}, & \tau_{0}=\left(\frac{(r-s) s d_{2}}{(r-q) q}\right)^{1 /(q-s)} .
\end{array}
$$

Proof. Let $(u, v) \in \mathscr{N}^{0}$. By (18) and (6),

$$
\begin{aligned}
& (p-s)\|u\|_{p}^{p}+(q-s)\|v\|_{q}^{q} \leq(r-s) d_{1}\left(\|u\|_{p}^{r}+\|v\|_{q}^{r}\right) \\
& (r-p)\|u\|_{p}^{p}+(r-q)\|v\|_{q}^{q} \leq(r-s) d_{2}\left(\|u\|_{p}^{s}+\|v\|_{q}^{s}\right) .
\end{aligned}
$$

If $\|v\|_{q}=0$, then it follows from (28)-(29) that

$$
\left(\frac{p-s}{(r-s) d_{1}}\right)^{1 /(r-p)} \leq\|u\|_{p} \leq\left(\frac{(r-s) d_{2}}{r-p}\right)^{1 /(p-s)}
$$

which contradicts (23).

Similarly, $\|u\|_{p}=0$ is impossible.

In view of (29), we have either

$$
(r-p)\|u\|_{p}^{p} \leq(r-s) d_{2}\|u\|_{p}^{s}
$$

or

$$
(r-q)\|v\|_{q}^{q} \leq(r-s) d_{2}\|v\|_{q}^{s}
$$

is satisfied. In the following, two cases are considered.

Case 1. Suppose that (31) holds. Then

$$
\|u\|_{p} \leq\left(\frac{(r-s) d_{2}}{r-p}\right)^{1 /(p-s)} .
$$

By (23), we obtain

$$
\left(\frac{(r-s) d_{2}}{r-p}\right)^{1 /(p-s)} \leq\left(\frac{p-s}{(r-s) d_{1}}\right)^{1 /(r-p)}
$$

and so

$$
\|u\|_{p} \leq\left(\frac{p-s}{(r-s) d_{1}}\right)^{1 /(r-p)}
$$

The last inequality is equivalent to

$$
(p-s)\|u\|_{p}^{p} \geq(r-s) d_{1}\|u\|_{p}^{r} .
$$

Hence, by (28), we infer that

$$
(q-s)\|v\|_{q}^{q} \leq(r-s) d_{1}\|v\|_{q}^{r},
$$

which is equivalent to

$$
\|v\|_{q} \geq\left(\frac{q-s}{(r-s) d_{1}}\right)^{1 /(r-q)}=v_{0} .
$$

On the other hand, we obtain from (29) that

$$
\phi\left(\|u\|_{p}\right) \leq \psi\left(\|v\|_{q}\right),
$$

where

$$
\begin{gathered}
\phi(t)=(r-p) t^{p}-(r-s) d_{2} t^{s}, \\
\psi(\tau)=(r-s) d_{2} \tau^{s}-(r-q) \tau^{q} .
\end{gathered}
$$


Obviously, $\phi(t)$ attains its minimum at $t_{0}$ and $\phi(\tau)$ attains its maximum at $\tau_{0}$. Furthermore, $\phi(t)$ is increasing on $\left(t_{0},+\infty\right), \psi(t)$ is decreasing on $\left(\tau_{0},+\infty\right)$, and

$$
\begin{aligned}
& \phi\left(t_{0}\right)=-\frac{(p-s)(r-p)}{s} t_{0}^{p}, \\
& \psi\left(\tau_{0}\right)=\frac{(q-s)(r-s) d_{2}}{q} \tau_{0}^{s} .
\end{aligned}
$$

In view of (24)-(25), we have $v_{0}>\tau_{0}$ and $\psi\left(v_{0}\right)<\phi\left(t_{0}\right)$ and so

$$
\psi\left(\|v\|_{q}\right) \leq \psi\left(v_{0}\right)<\phi\left(t_{0}\right)
$$

which contradicts (39). Thus $\mathcal{N}^{0}=\emptyset$.

Case 2. Suppose that (32) holds. Then, by (24),

$$
\|v\|_{q} \leq\left(\frac{(r-s) d_{2}}{r-q}\right)^{1 /(q-s)} \leq\left(\frac{q-s}{(r-s) d_{1}}\right)^{1 /(r-q)},
$$

which is equivalent to

$$
(q-s)\|v\|_{q}^{q} \geq(r-s) d_{1}\|v\|_{q}^{r} .
$$

Thus (28) and (44) imply

$$
(p-s)\|u\|_{p}^{p} \leq(r-s) d_{1}\|u\|_{p}^{r} .
$$

Hence

$$
\|u\|_{p} \geq\left(\frac{p-s}{(r-s) d_{1}}\right)^{1 /(r-p)}=u_{0} .
$$

In view of (23) and (26), we obtain $u_{0}>t_{0}, \phi\left(u_{0}\right)>\psi\left(\tau_{0}\right)$ and so $\phi\left(\|u\|_{p}\right) \geq \phi\left(u_{0}\right)>\psi\left(\tau_{0}\right)$, which contradicts (39). Thus $\mathcal{N}^{0}=\emptyset$.

In order to get a better understanding of the Nehari manifold, we consider the function $m_{u, v}(t)$ defined by

$$
m_{(u, v)}(t)=t^{p-r}\|u\|_{p}^{p}+t^{q-r}\|v\|_{q}^{q}-s t^{s-r} c(u, v)
$$

for $t>0$, where $c(u, v)$ is defined in (4). Obviously, $\lim _{t \rightarrow 0^{+}} m_{(u, v)}(t)=-\infty, \lim _{t \rightarrow+\infty} m_{(u, v)}(t)=0$, and

$$
\begin{gathered}
m_{(u, v)}^{\prime}(t) \\
=t^{s-r-1}\left((p-r) t^{p-s}\|u\|_{p}^{p}+(q-r) t^{q-s}\|v\|_{q}^{q}\right. \\
-s(s-r) c(u, v)) .
\end{gathered}
$$

If $c(u, v)>0$, then there is unique $t_{1}=t_{1}(u, v)$ such that $m_{(u, v)}^{\prime}\left(t_{1}\right)=0$. Furthermore, $m_{(u, v)}^{\prime}(t)>0$ for $t \in\left(0, t_{1}\right)$ and $m_{(u, v)}^{\prime}(t)<0$ for $t \in\left(t_{1}, \infty\right)$. By direct computation, we can deduce that

$$
\begin{aligned}
m_{(u, v)}\left(t_{1}\right) & =t_{1}^{s-r}\left(t_{1}^{p-s}\|u\|_{p}^{p}+t_{1}^{q-s}\|v\|_{q}^{q}-s c(u, v)\right) \\
& =\frac{p-s}{r-s} t_{1}^{p-r}\|u\|_{p}^{p}+\frac{q-s}{r-s} t_{1}^{q-r}\|v\|_{q}^{q} .
\end{aligned}
$$

Moreover

$$
\begin{aligned}
0<t_{1}(u, v)<\min \{ & \left(\frac{s(r-s) c(u, v)}{(r-p)\|u\|_{p}^{p}}\right)^{1 /(p-s)}, \\
& \left.\left(\frac{s(r-s) c(u, v)}{(r-q)\|v\|_{q}^{q}}\right)^{1 /(q-s)}\right\}
\end{aligned}
$$

if $\|u\|_{p}>0$ and $\|v\|_{q}>0$. Then the following lemma holds.

Lemma 4. For each $(u, v) \in X \backslash\{(0,0)\}$ with $c(u, v)>0$, one has $m_{(u, v)}\left(t_{1}(u, v)\right)>r b(u, v)$ provided

$$
\begin{aligned}
& d_{1}^{p-s} d_{2}^{r-p} \leq 2^{s-r}(p-s)^{p-s}(r-p)^{r-p}(r-s)^{s-r}, \\
& d_{1}^{q-s} d_{2}^{r-q} \leq 2^{s-r}(q-s)^{q-s}(r-q)^{r-q}(r-s)^{s-r} .
\end{aligned}
$$

Proof. The proof is divided into the following four cases.

Case $1\left(\|u\|_{p}=0<\|v\|_{q}\right)$. In this case,

$$
t_{1}=\left(\frac{s(r-s) c(u, v)}{(r-q)\|v\|_{q}^{q}}\right)^{1 /(q-s)} .
$$

Thus, by (6), we infer that

$$
\begin{aligned}
m_{(u, v)}\left(t_{1}\right) & \\
= & \frac{q-s}{r-s}\left(\frac{s(r-s) c(u, v)}{(r-q)\|v\|_{q}^{q}}\right)^{(q-r) /(q-s)}\|v\|_{q}^{q} \\
\geq & \frac{q-s}{r-s}\left(\frac{(r-s) d_{2}}{r-q}\right)^{(q-r) /(q-s)}\|v\|_{q}^{r} \\
\geq & \frac{q-s}{r-s}\left(\frac{(r-s) d_{2}}{r-q}\right)^{(q-r) /(q-s)} \frac{r b(u, v)}{d_{1}} .
\end{aligned}
$$

In view of (52), we have

$$
\frac{q-s}{r-s}\left(\frac{(r-s) d_{2}}{r-q}\right)^{(q-r) /(q-s)} \frac{1}{d_{1}}>1
$$

and so $m_{(u, v)}\left(t_{1}\right)>r b(u, v)$.

Case $2\left(\|v\|_{q}=0<\|u\|_{p}\right)$. Then

$$
t_{1}=\left(\frac{s(r-s) c(u, v)}{(r-p)\|u\|_{p}^{p}}\right)^{1 /(p-s)}
$$

and so

$$
\begin{aligned}
m_{(u, v)}\left(t_{1}\right) & =\frac{p-s}{r-s}\left(\frac{s(r-s) c(u, v)}{(r-p)\|u\|_{p}^{p}}\right)^{(p-r) /(p-s)}\|u\|_{p}^{p} \\
& \geq \frac{p-s}{r-s}\left(\frac{(r-s) d_{2}}{r-p}\right)^{(p-r) /(p-s)}\|u\|_{p}^{r} \\
& \geq \frac{p-s}{r-s}\left(\frac{(r-s) d_{2}}{r-p}\right)^{(p-r) /(p-s)} \frac{r b(u, v)}{d_{1}} .
\end{aligned}
$$

Thus $m_{(u, v)}\left(t_{1}\right)>r b(u, v)$ follows from (51). 
Case $3\left(0<\|u\|_{p} \leq\|v\|_{q}\right)$. It follows from (49), (50), and (6) that

$$
\begin{aligned}
& m_{(u, v)}\left(t_{1}\right) \\
&> \frac{p-s}{r-s}\left(\frac{s(r-s) c(u, v)}{(r-p)\|u\|_{p}^{p}}\right)^{(p-r) /(p-s)}\|u\|_{p}^{p} \\
&+\frac{q-s}{r-s}\left(\frac{s(r-s) c(u, v)}{(r-q)\|v\|_{q}^{q}}\right)^{(q-r) /(q-s)}\|v\|_{q}^{q} \\
& \geq \frac{p-s}{r-s}\left(\frac{(r-s) d_{2}\left(\|u\|_{p}^{s}+\|v\|_{q}^{s}\right)}{(r-p)\|u\|_{p}^{p}}\right) \\
&+ \frac{q-s}{r-s}\left(\frac{(r-s) d_{2}\left(\|u\|_{p}^{s}+\|v\|_{q}^{s}\right)}{(r-q)\|v\|_{q}^{q}}\right)^{(q-r) /(q-s)}\|u\|_{p}^{p} \\
&
\end{aligned}
$$

Since $\|u\|_{p} \leq\|v\|_{q}$, we have

$$
\|u\|_{p}^{s}+\|v\|_{q}^{s} \leq 2\|v\|_{q}^{s}, \quad\|u\|_{p}^{r}+\|v\|_{q}^{r} \leq 2\|v\|_{q}^{r} .
$$

Thus

$$
\begin{aligned}
m_{(u, v)}\left(t_{1}\right) & >\frac{q-s}{r-s}\left(\frac{2(r-s) d_{2}\|v\|_{q}^{s}}{(r-q)\|v\|_{q}^{q}}\right)^{(q-r) /(q-s)}\|v\|_{q}^{q} \\
& =\frac{q-s}{r-s}\left(\frac{2(r-s) d_{2}}{r-q}\right)^{(q-r) /(q-s)}\|v\|_{q}^{r} \\
& \geq \frac{q-s}{r-s}\left(\frac{2(r-s) d_{2}}{r-q}\right)^{(q-r) /(q-s)} \frac{r b(u, v)}{2 d_{1}} .
\end{aligned}
$$

Hence $m_{(u, v)}\left(t_{1}\right)>r b(u, v)$ provided

$$
\frac{q-s}{r-s}\left(\frac{2(r-s) d_{2}}{r-q}\right)^{(q-r) /(q-s)} \frac{1}{2 d_{1}} \geq 1
$$

which is equivalent to (52).

Case $4\left(\|u\|_{p}>\|v\|_{q}>0\right)$. In this case, (58) still holds. Thus, in view of (6), we infer

$$
\begin{aligned}
m_{(u, v)}\left(t_{1}\right) & >\frac{p-s}{r-s}\left(\frac{2(r-s) d_{2}\|u\|_{p}^{s}}{(r-p)\|u\|_{p}^{p}}\right)^{(p-r) /(p-s)}\|u\|_{p}^{p} \\
& \geq \frac{p-s}{r-s}\left(\frac{2(r-s) d_{2}}{r-p}\right)^{(p-r) /(p-s)}\|u\|_{p}^{r} \\
& \geq \frac{p-s}{r-s}\left(\frac{2(r-s) d_{2}}{r-p}\right)^{(p-r) /(p-s)} \frac{r b(u, v)}{2 d_{1}} .
\end{aligned}
$$

Hence $m_{(u, v)}\left(t_{1}\right)>r b(u, v)$ follows from $(51)$.
By Lemma 4, we have the following.

Lemma 5. Suppose that (51)-(52) hold. Then, for each $(u, v) \in$ $X \backslash\{(0,0)\}$ with $c(u, v)>0$, one has the following.

(i) If $b(u, v) \leq 0$, then there is unique $t^{+} \in\left(0, t_{1}\right)$ such that $J(t u, t v)$ is decreasing on $\left(0, t^{+}\right)$and increasing on $\left(t^{+}, \infty\right)$. Moreover $\left(t^{+} u, t^{+} v\right) \in \mathcal{N}^{+}$and

$$
J\left(t^{+} u, t^{+} v\right)=\inf _{t \geq 0} J(t u, t v)
$$

(ii) If $b(u, v)>0$, then there are $t^{+}$and $t^{-}$with $0<$ $t^{+}<t_{1}<t^{-}$such that $J(t u, t v)$ is decreasing on $\left(0, t^{+}\right)$, increasing on $\left(t^{+}, t^{-}\right)$, and decreasing on $\left(t^{-}, \infty\right)$. Moreover $\left(t^{+} u, t^{+} v\right) \in \mathcal{N}^{+},\left(t^{-} u, t^{-} v\right) \in \mathcal{N}^{-}$, and

$$
\begin{aligned}
& J\left(t^{+} u, t^{+} v\right)=\inf _{0 \leq t \leq t^{-}} J(t u, t v), \\
& J\left(t^{-} u, t^{-} v\right)=\sup _{t \geq t^{+}} J(t u, t v) .
\end{aligned}
$$

Proof. Fix $(u, v) \in X \backslash\{(0,0)\}$ with $c(u, v)>0$. By (17) and (19), we infer that

$$
\begin{aligned}
& \Phi(t u, t v) \\
& =t^{p}\|u\|_{p}^{p}+t^{q}\|v\|_{q}^{q}-t^{r} r b(u, v)-t^{s} s c(u, v) \\
& =t^{r}\left(m_{(u, v)}(t)-r b(u, v)\right), \\
& \left\langle\Phi^{\prime}(t u, t v),(t u, t v)\right\rangle \\
& =(p-r) t^{p}\|u\|_{p}^{p}+(q-r) t^{q}\|v\|_{q}^{q} \\
& \quad-(s-r) t^{s} s c(u, v)=t^{r+1} m_{(u, v)}^{\prime}(t) .
\end{aligned}
$$

(i) Suppose that $b(u, v) \leq 0$. Then $m_{(u, v)}(t)=r b(u, v)$ has unique solution $t^{+} \in\left(0, t_{1}\right)$ and $m_{(u, v)}^{\prime}\left(t^{+}\right)>0$. Hence $\Phi\left(t^{+} u, t^{+} v\right)=\left(t^{+}\right)^{r}\left(m_{(u, v)}\left(t^{+}\right)-r b(u, v)\right)=0$, $\left\langle\Phi^{\prime}\left(t^{+} u, t^{+} v\right),\left(t^{+} u, t^{+} v\right)\right\rangle=\left(t^{+}\right)^{r+1} m_{(u, v)}^{\prime}\left(t^{+}\right)>0$, and so $\left(t^{+} u, t^{+} v\right) \in \mathcal{N}^{+}$. Moreover, since

$$
\begin{aligned}
\frac{d}{d t} J(t u, t v)= & t^{p-1}\|u\|_{p}^{p}+t^{q-1}\|v\|_{q}^{q}-t^{r-1} r b(u, v) \\
& -t^{s-1} s c(u, v)=\frac{1}{t} \Phi(t u, t v),
\end{aligned}
$$

we obtain $(d / d t) J(t u, t v)<0$ for $t \in\left(0, t^{+}\right)$and $(d / d t) J(t u, t v)>0$ for $t>t^{+}$. Thus $J(t u, t v)$ is decreasing on $\left(0, t^{+}\right)$and increasing on $\left(t^{+}, \infty\right)$ and $J\left(t^{+} u, t^{+} v\right)=\inf _{t \geq 0} J(t u, t v)$.

(ii) Suppose that $b(u, v)>0$. Since $m_{(u, v)}\left(t_{1}\right)>r b(u, v)$, the equation $m_{(u, v)}(t)=r b(u, v)$ has exactly two solutions $t^{+}$, $t^{-}$with $0<t^{+}<t_{1}<t^{-}$such that $m_{(u, v)}^{\prime}\left(t^{-}\right)<0<m_{(u, v)}^{\prime}\left(t^{+}\right)$. Thus there are exactly two 
multiples of $u$ lying in $\mathcal{N}$; namely, $\left(t^{+} u, t^{+} v\right) \in \mathscr{N}^{+}$ and $\left(t^{-} u, t^{-} v\right) \in \mathcal{N}^{-}$. Since

$$
\frac{d}{d t} J(t u, t v) \begin{cases}<0, & 0<t<t^{+} \\ >0, & t^{+}<t<t^{-} \\ <0, & t>t^{-}\end{cases}
$$

we have $J(t u, t v)$ is decreasing on $\left(0, t^{+}\right)$, increasing on $\left(t^{+}, t^{-}\right)$, and decreasing on $\left(t^{-}, \infty\right)$ and $(64)$ holds.

Similarly, we define

$$
n_{(u, v)}(t)=t^{p-s}\|u\|_{p}^{p}+t^{q-s}\|v\|_{q}^{q}-t^{r-s} r b(u, v), \quad \text { for } t>0
$$

where $b(u, v)$ is defined in (4). Clearly $\lim _{t \rightarrow 0^{+}} n_{(u, v)}(t)=$ $0, \lim _{t \rightarrow \infty} n_{(u, v)}(t)=-\infty$, and

$$
\begin{aligned}
n_{(u, v)}^{\prime}(t)= & (p-s) t^{p-s-1}\|u\|_{p}^{p}+(q-s) t^{q-s-1}\|v\|_{q}^{q} \\
& -(r-s) t^{r-s-1} r b(u, v) .
\end{aligned}
$$

If $b(u, v)>0$, then there is unique $t_{2}=t_{2}(u, v)$ such that $n_{(u, v)}^{\prime}\left(t_{2}\right)=0$. Furthermore, $n_{(u, v)}^{\prime}(t)>0$ for $0<t<t_{2}$ and $n_{(u, v)}^{\prime}(t)<0$ for $t>t_{2}$.

Using arguments similar to those in the proof of Lemmas 4 and 5 , we have the following.

Lemma 6. For each $(u, v) \in X \backslash\{(0,0)\}$ with $b(u, v)>0$, one has $n_{(u, v)}\left(t_{2}(u, v)\right)>s c(u, v)$ provided (51)-(52) hold.

Lemma 7. Suppose that (51)-(52) hold. Then, for each $(u, v) \in$ $X \backslash\{(0,0)\}$ with $b(u, v)>0$, one has the following.

(i) If $c(u, v) \leq 0$, then there is unique $t^{-} \in\left(t_{2}, \infty\right)$ such that $J(t u, t v)$ is increasing on $\left(0, t^{-}\right)$and decreasing on $\left(t^{-}, \infty\right)$. Moreover $\left(t^{-} u, t^{-} v\right) \in \mathcal{N}^{-}$and

$$
J\left(t^{-} u, t^{-} v\right)=\sup _{t \geq 0} J(t u, t v) .
$$

(ii) If $c(u, v)>0$, then there are $t^{+}$and $t^{-}$with $0<t^{+}<t_{2}$ $<t^{-}$such that $J(t u, t v)$ is decreasing on $\left(0, t^{+}\right)$, increasing on $\left(t^{+}, t^{-}\right)$, and decreasing on $\left(t^{-}, \infty\right)$. Moreover $\left(t^{+} u, t^{+} v\right) \in \mathcal{N}^{+},\left(t^{-} u, t^{-} v\right) \in \mathcal{N}^{-}$, and

$$
\begin{aligned}
& J\left(t^{+} u, t^{+} v\right)=\inf _{0 \leq t \leq t^{-}} J(t u, t v), \\
& J\left(t^{-} u, t^{-} v\right)=\sup _{t \geq t^{+}} J(t u, t v) .
\end{aligned}
$$

\section{Existence of Nonzero Solutions}

In this section, we will give simple proofs of the existence of two nonzero weak solutions, one in $\mathscr{N}^{+}$and one in $\mathcal{N}^{-}$.

Proposition 8. Suppose that (25)-(26) and (51)-(52) hold. Then there exists a minimizer $\left(u_{0}^{+}, v_{0}^{+}\right)$of $J$ on $\mathcal{N}^{+}$and $\left(u_{0}^{+}, v_{0}^{+}\right) \not \equiv(0,0)$.
Proof. Since $J$ is bounded below on $\mathcal{N}$ and so on $\mathcal{N}^{+}$, there exists a minimizing sequence $\left\{\left(u_{n}, v_{n}\right)\right\} \subset \mathcal{N}^{+}$such that

$$
\lim _{n \rightarrow \infty} J\left(u_{n}, v_{n}\right)=\inf _{(u, v) \in \mathscr{N}^{+}} J(u, v) .
$$

Since $J$ is coercive, $\left\{\left(u_{n}, v_{n}\right)\right\}$ is bounded in $X$. Thus we may assume, without loss of generality, that $\left(u_{n}, v_{n}\right) \rightarrow$ $\left(u_{0}^{+}, v_{0}^{+}\right)$weakly in $X$. By $\left(\mathrm{H}_{3}\right)$,

$$
\begin{aligned}
& \lim _{n \rightarrow \infty} b\left(u_{n}, v_{n}\right)=b\left(u_{0}^{+}, v_{0}^{+}\right), \\
& \lim _{n \rightarrow \infty} c\left(u_{n}, v_{n}\right)=c\left(u_{0}^{+}, v_{0}^{+}\right) .
\end{aligned}
$$

Since $\left(u_{n}, v_{n}\right) \in \mathcal{N}^{+}$, we can infer from (18) that

$$
(p-s)\left\|u_{n}\right\|_{p}^{p}+(q-s)\left\|v_{n}\right\|_{q}^{q}>(r-s) r b\left(u_{n}, v_{n}\right) .
$$

Then

$$
\begin{aligned}
J\left(u_{n}, v_{n}\right) & \\
= & \left(\frac{1}{p}-\frac{1}{s}\right)\left\|u_{n}\right\|_{p}^{p}+\left(\frac{1}{q}-\frac{1}{s}\right)\left\|v_{n}\right\|_{q}^{q} \\
& -\left(\frac{1}{r}-\frac{1}{s}\right) r b\left(u_{n}, v_{n}\right)<\left(\frac{1}{p}-\frac{1}{s}\right)\left\|u_{n}\right\|_{p}^{p} \\
& +\left(\frac{1}{q}-\frac{1}{s}\right)\left\|v_{n}\right\|_{q}^{q}+\frac{1}{r s}\left((p-s)\left\|u_{n}\right\|_{p}^{p}+(q-s)\|v\|_{q}^{q}\right) \\
= & -\frac{(r-p)(p-s)}{r p s}\left\|u_{n}\right\|_{p}^{p}-\frac{(r-q)(q-s)}{r q s}\left\|v_{n}\right\|_{q}^{q}<0 .
\end{aligned}
$$

This implies

$$
\lim _{n \rightarrow \infty} J\left(u_{n}, v_{n}\right)=\inf _{(u, v) \in \mathscr{N}^{+}} J(u, v)<0 .
$$

Notice that

$$
\begin{aligned}
& J\left(u_{n}, v_{n}\right) \\
& \quad=\left(\frac{1}{p}-\frac{1}{r}\right)\left\|u_{n}\right\|_{p}^{p}+\left(\frac{1}{q}-\frac{1}{r}\right)\left\|v_{n}\right\|_{q}^{q}-\left(\frac{1}{s}-\frac{1}{r}\right) s c\left(u_{n}, v_{n}\right)
\end{aligned}
$$

and, letting $n \rightarrow+\infty$, we obtain $c\left(u_{0}^{+}, v_{0}^{+}\right)>0$. Hence $\left(u_{0}^{+}, v_{0}^{+}\right) \not \equiv(0,0)$.

Now we prove that $\left(u_{n}, v_{n}\right) \rightarrow\left(u_{0}^{+}, v_{0}^{+}\right)$strongly in $X$. Suppose otherwise; then

$$
\left\|u_{0}^{+}\right\|_{p}^{p}+\left\|v_{0}^{+}\right\|_{q}^{q}<\liminf _{n \rightarrow \infty}\left(\left\|u_{n}\right\|_{p}^{p}+\left\|v_{n}\right\|_{q}^{q}\right) .
$$

Let $t_{1}\left(u_{n}, v_{n}\right)$ be the unique solution of $m_{\left(u_{n}, v_{n}\right)}^{\prime}(t)=0$ and let $t_{1}\left(u_{0}^{+}, v_{0}^{+}\right)$be the unique solution of $m_{\left(u_{0}^{+}, v_{0}^{+}\right)}^{\prime}(t)=0$, where $m_{(u, v)}(t)$ is defined by (47). Since $\left(u_{n}, v_{n}\right) \in \mathcal{N}^{+}$, we have from (19) that

$$
(r-s) s c\left(u_{n}, v_{n}\right)>(r-p)\left\|u_{n}\right\|_{p}^{p}+(r-q)\left\|v_{n}\right\|_{q}^{q}
$$

and so $m_{\left(u_{n}, v_{n}\right)}^{\prime}(1)>0$. Thus $t_{1}\left(u_{n}, v_{n}\right)>1$. 
Since $c\left(u_{0}^{+}, v_{0}^{+}\right)>0$, it follows from Lemma 5 that there is unique $t_{0}^{+} \in\left(0, t_{1}\left(u_{0}^{+}, v_{0}^{+}\right)\right)$such that

$$
\begin{gathered}
\left(t_{0}^{+} u_{0}^{+}, t_{0}^{+} v_{0}^{+}\right) \in \mathcal{N}^{+} \\
J\left(t_{0}^{+} u_{0}^{+}, t_{0}^{+} v_{0}^{+}\right)=\inf _{0 \leq t \leq t_{1}\left(u_{0}^{+}, v_{0}^{+}\right)} J(t u, t v) .
\end{gathered}
$$

From (79), we have

$$
0=\Phi\left(t_{0}^{+} u_{0}^{+}, t_{0}^{+} v_{0}^{+}\right)<\liminf _{n \rightarrow \infty} \Phi\left(t_{0}^{+} u_{n}, t_{0}^{+} v_{n}\right) .
$$

This implies $\Phi\left(t_{0}^{+} u_{n}, t_{0}^{+} v_{n}\right)>0$ for $n$ sufficiently large.

Since $m_{\left(u_{n}, v_{n}\right)}(t)$ is increasing on $\left(0, t_{1}\left(u_{n}, v_{n}\right)\right)$ and so on $(0,1), \Phi\left(t u_{n}, t v_{n}\right)$ is also increasing on $(0,1)$.

In view of $\Phi\left(u_{n}, v_{n}\right)=0$ and $\Phi\left(t_{0}^{+} u_{n}, t_{0}^{+} v_{n}\right)>0$ for $n$ sufficiently large, we deduce that $t_{0}^{+}>1$ and so

$$
J\left(t_{0}^{+} u_{0}^{+}, t_{0}^{+} v_{0}^{+}\right)<J\left(u_{0}^{+}, v_{0}^{+}\right)<\lim _{n \rightarrow \infty} J\left(u_{n}, v_{n}\right),
$$

a contradiction. Hence $\left(u_{n}, v_{n}\right) \rightarrow\left(u_{0}^{+}, v_{0}^{+}\right)$strongly in $X$. This implies $J\left(u_{n}, v_{n}\right) \rightarrow J\left(u_{0}^{+}, v_{0}^{+}\right)$as $n \rightarrow \infty$. Thus $\left(u_{0}^{+}, v_{0}^{+}\right)$ is a minimizer for $J$ on $\mathcal{N}^{+}$.

Proposition 9. Suppose that (25)-(26) and (51)-(52) hold. Then there exists a minimizer $\left(u_{0}^{-}, v_{0}^{-}\right)$of $J$ in $\mathcal{N}^{-}$and $\left(u_{0}^{-}, v_{0}^{-}\right) \not \equiv(0,0)$.

Proof. Similarly as the proof of Proposition 8, there exists a minimizing sequence $\left\{\left(u_{n}, v_{n}\right)\right\} \subset \mathcal{N}^{-}$and $\left(u_{0}^{-}, v_{0}^{-}\right) \in X$ such that

$$
\begin{array}{r}
\lim _{n \rightarrow \infty} J\left(u_{n}, v_{n}\right)=\inf _{(u, v) \in \mathcal{N}^{-}} J(u, v), \\
\left(u_{n}, v_{n}\right) \rightarrow\left(u_{0}^{-}, v_{0}^{-}\right) \quad \text { weakly in } X, \\
b\left(u_{n}, v_{n}\right) \longrightarrow b\left(u_{0}^{-}, v_{0}^{-}\right), \quad c\left(u_{n}, v_{n}\right) \longrightarrow c\left(u_{0}^{-}, v_{0}^{-}\right) \\
\text {strongly in } X .
\end{array}
$$

Since $\left(u_{n}, v_{n}\right) \in \mathcal{N}^{-}$, we can infer from (18) and (6) that

$$
\begin{aligned}
(p-s)\left\|u_{n}\right\|_{p}^{p}+(q-s)\left\|v_{n}\right\|_{q}^{q} & <(r-s) r b\left(u_{n}, v_{n}\right) \\
& \leq(r-s) d_{1}\left(\left\|u_{n}\right\|_{p}^{r}+\left\|v_{n}\right\|_{q}^{r}\right) .
\end{aligned}
$$

Then either

$$
(p-s)\left\|u_{n}\right\|_{p}^{p}<(r-s) d_{1}\left\|u_{n}\right\|_{p}^{r}
$$

or

$$
(q-s)\left\|v_{n}\right\|_{q}^{q}<(r-s) d_{1}\left\|v_{n}\right\|_{q}^{r}
$$

is satisfied. That is to say, either $\left\|u_{n}\right\|_{p}>u_{0}$ or $\left\|v_{n}\right\|_{q}>v_{0}$ is satisfied, where $u_{0}$ and $v_{0}$ are defined in Lemma 3 . Hence, for all $n$, we obtain from (85) that

$$
(r-s) r b\left(u_{n}, v_{n}\right)>\min \left\{(p-s) u_{0}^{p},(q-s) v_{0}^{q}\right\} .
$$

Letting $n \rightarrow+\infty$, we get that

$$
(r-s) r b\left(u_{0}^{-}, v_{0}^{-}\right) \geq \min \left\{(p-s) u_{0}^{p},(q-s) v_{0}^{q}\right\}>0 .
$$

Hence $b\left(u_{0}^{-}, v_{0}^{-}\right)>0$ and so $\left(u_{0}^{-}, v_{0}^{-}\right) \not \equiv(0,0)$.

Now we prove that $\left(u_{n}, v_{n}\right) \rightarrow\left(u_{0}^{-}, v_{0}^{-}\right)$strongly in $X$. Suppose on the contrary; then

$$
\left\|u_{0}^{-}\right\|_{p}^{p}+\left\|v_{0}^{-}\right\|_{q}^{q}<\liminf _{n \rightarrow \infty}\left(\left\|u_{n}\right\|_{p}^{p}+\left\|v_{n}\right\|_{q}^{q}\right) .
$$

Let $t_{2}\left(u_{n}, v_{n}\right)$ be the unique solution of $n_{\left(u_{n}, v_{n}\right)}^{\prime}(t)=0$ and let $t_{2}\left(u_{0}^{-}, v_{0}^{-}\right)$be the unique solution of $n_{\left(u_{0}^{-}, v_{0}^{-}\right)}^{\prime}(t)=0$, where $n_{(u, v)}(t)$ is defined by $(69)$.

By Lemma 7 , there is unique $t_{0}^{-}>t_{2}\left(u_{0}^{-}, v_{0}^{-}\right)$such that $\left(t_{0}^{-} u_{0}^{-}, t_{0}^{-} v_{0}^{-}\right) \in \mathcal{N}^{-}$. In view of (90), we infer that

$$
\liminf _{n \rightarrow \infty} \Phi\left(t_{0}^{-} u_{n}, t_{0}^{-} v_{n}\right)>\Phi\left(t_{0}^{-} u_{0}^{-}, t_{0}^{-} v_{0}^{-}\right)=0 .
$$

This implies $\Phi\left(t_{0}^{-} u_{n}, t_{0}^{-} v_{n}\right)>0$ for $n$ sufficiently large.

Since $\left(u_{n}, v_{n}\right) \in \mathcal{N}^{-}, \Phi\left(u_{n}, v_{n}\right)=0$, and it is clear from Lemmas 5 and 7 that $J(t u, t v)$ is increasing on $\left(t_{0}^{-}, 1\right)$; that is, $J\left(t_{0}^{-} u_{n}, t_{0}^{-} v_{n}\right)<J\left(u_{n}, v_{n}\right)$.

Thus

$$
J\left(t_{0}^{-} u_{0}^{-}, t_{0}^{-} v_{0}^{-}\right)<\lim _{n \rightarrow \infty} J\left(t_{0}^{-} u_{n}, t_{0}^{-} v_{n}\right) \leq \lim _{n \rightarrow \infty} J\left(u_{n}, v_{n}\right),
$$

which is a contradiction. Hence $\left(u_{n}, v_{n}\right) \rightarrow\left(u_{0}^{-}, v_{0}^{-}\right)$strongly in $X$. This implies $J\left(u_{n}, v_{n}\right) \rightarrow J\left(u_{0}^{-}, v_{0}^{-}\right)$as $n \rightarrow \infty$. Thus $\left(u_{0}^{-}, v_{0}^{-}\right)$is a minimizer for $J$ on $\mathcal{N}^{-}$.

By Propositions 8 and 9, we can prove our main result read as follows.

Theorem 10. Problem (3) has at least two solutions $\left(u_{0}^{+}, v_{0}^{+}\right)$ and $\left(u_{0}^{-}, v_{0}^{-}\right)$such that $\left(u_{0}^{ \pm}, v_{0}^{ \pm}\right) \neq \equiv(0,0)$ if $(25),(26),(51)$, and (52) are satisfied.

Proof. By Propositions 8 and 9, there exist $\left(u_{0}^{+}, v_{0}^{+}\right) \in \mathcal{N}^{+}$ and $\left(u_{0}^{-}, v_{0}^{-}\right) \in \mathcal{N}^{-}$such that $J\left(u_{0}^{+}, v_{0}^{+}\right)=\inf _{(u, v) \in \mathcal{N}^{+}} J(u, v)$, $J\left(u_{0}^{-}, v_{0}^{-}\right)=\inf _{(u, v) \in \mathcal{N}^{-}} J(u, v)$, and $\left(u_{0}^{ \pm}, v_{0}^{ \pm}\right) \neq 0$. By Lemma 2, $\left(u_{0}^{ \pm}, v_{0}^{ \pm}\right)$are critical points of $J$ on $X$ and hence are weak solutions of (3).

Remark 11. Inequalities (25), (26), (51), and (52) can be fulfilled provided that $d_{1}$ or $d_{2}$ is sufficiently small.

\section{Applications}

In this section, we give some applications of Theorem 10.

(I) We consider the multiplicity of nonnegative solutions for the following $(p, q)$-Laplacian system with nonlinear boundary conditions:

$$
\begin{gathered}
-\Delta_{p} u+|u|^{p-2} u=\frac{\alpha}{\alpha+\beta} f(x)|u|^{\alpha-2} u|v|^{\beta}, \quad \text { in } \Omega, \\
-\Delta_{q} v+|v|^{q-2} v=\frac{\beta}{\alpha+\beta} f(x)|u|^{\alpha}|v|^{\beta-2} v, \quad \text { in } \Omega, \\
\frac{\partial u}{\partial n}=\lambda g(x)|u|^{\gamma-2} u, \quad \frac{\partial v}{\partial n}=\mu h(x)|v|^{\gamma-2} v, \quad \text { on } \partial \Omega,
\end{gathered}
$$


where $\alpha, \beta, \gamma>1, \Omega \subset \mathbb{R}^{N}$ is a bounded domain with smooth boundary, $\lambda, \mu$ are parameters in $\mathbb{R} \backslash\{0\}$, and the weight functions $f \in C(\bar{\Omega})$ and $g, h \in C(\partial \Omega)$ satisfy the following condition:

$\left(\mathrm{A}_{1}\right) f^{+}=\max \{f, 0\} \not \equiv \quad 0, g^{ \pm}=\max \{ \pm g, 0\} \quad \equiv \quad 0$, and $h^{ \pm}=\max \{ \pm h, 0\} \quad \equiv 0$.

We consider problem (93) in the framework of the Sobolev space $X=W^{1, p}(\Omega) \times W^{1, q}(\Omega)$. A pair of functions $(u, v) \in X$ is said to be a weak solution of problem (93) if

$$
\begin{aligned}
& \int_{\Omega}\left(|\nabla u|^{p-2} \nabla u \nabla \phi_{1}+|u|^{p-2} u \phi_{1}\right) d x-\lambda \int_{\partial \Omega} g|u|^{\gamma-2} u \phi_{1} d s \\
& -\frac{\alpha}{\alpha+\beta} \int_{\Omega} f|u|^{\alpha-2} u|v|^{\beta} \phi_{1} d x, \\
& \int_{\Omega}\left(|\nabla v|^{q-2} \nabla v \nabla \phi_{2}+|v|^{q-2} v \phi_{2}\right) d x-\mu \int_{\partial \Omega} h|v|^{\gamma-2} v \phi_{2} d s \\
& \quad-\frac{\beta}{\alpha+\beta} \int_{\Omega} f|u|^{\alpha}|v|^{\beta-2} v \phi_{2} d x
\end{aligned}
$$

hold for all $\left(\phi_{1}, \phi_{2}\right) \in X$, where $d s$ is the measure on the boundary. In the following, two cases are considered.

Case $1\left(2<\alpha+\beta<p \leq q<\gamma<p_{*}\left(p_{*}=(N-1) p /(N-p)\right.\right.$ if $N>p, p_{*}=\infty$ if $\left.\left.N \leq p\right)\right)$. Let $\alpha+\beta=s, \gamma=r$. Then $\left(\mathrm{H}_{1}\right)$ is satisfied.

For all $(u, v),\left(\phi_{1}, \phi_{2}\right) \in X$, we define potential operators $B, C: X \rightarrow X^{*}$ given by

$$
\begin{aligned}
\left\langle B(u, v),\left(\phi_{1}, \phi_{2}\right)\right\rangle= & \lambda \int_{\partial \Omega} g|u|^{r-2} u \phi_{1} d s \\
& +\mu \int_{\partial \Omega} h|v|^{r-2} v \phi_{2} d s \\
\left\langle C(u, v),\left(\phi_{1}, \phi_{2}\right)\right\rangle= & \frac{\alpha}{\alpha+\beta} \int_{\Omega} f|u|^{\alpha-2} u|v|^{\beta} \phi_{1} d x \\
& +\frac{\beta}{\alpha+\beta} \int_{\Omega} f|u|^{\alpha}|v|^{\beta-2} v \phi_{2} d x .
\end{aligned}
$$

Then we have

$$
\begin{gathered}
r b(u, v)=\lambda \int_{\partial \Omega} g|u|^{r} d s+\mu \int_{\partial \Omega} h|v|^{r} d s, \\
s c(u, v)=\int_{\Omega} f|u|^{\alpha}|v|^{\beta} d x .
\end{gathered}
$$

By assumption $\left(A_{1}\right)$, we have $\left(H_{2}\right)$ is satisfied.

It is clear that the corresponding energy functional of (93) is defined by

$$
J(u, v)=\frac{1}{p}\|u\|_{p}^{p}+\frac{1}{q}\|v\|_{q}^{q}-b(u, v)-c(u, v) .
$$

Let $S_{\sigma, \tau}$ and $C_{\sigma, \tau}$ be the best Sobolev constant for the embedding of $W^{1, \tau}(\Omega) \hookrightarrow L^{\sigma}(\Omega)$ for $1 \leq \sigma<\tau^{*}$ and the best Sobolev trace constant for the embedding of $W^{1, \tau}(\Omega) \hookrightarrow$
$L^{\sigma}(\partial \Omega)$ for $1 \leq \sigma<\tau_{*}$, respectively, where $\tau^{*}=\tau N /(N-$ $\tau), \tau_{*}=\tau(N-1) /(N-\tau)$ if $N>\tau$ and $\tau^{*}=\tau_{*}=\infty$ if $N \leq \tau$. Then we have

$r b(u, v)$

$$
\begin{aligned}
& \leq|\lambda|\|g\|_{\infty} \int_{\partial \Omega}|u|^{r} d s+|\mu|\|h\|_{\infty} \int_{\partial \Omega}|v|^{r} d s \\
& \leq|\lambda|\|g\|_{\infty} C_{r, p}^{r}\|u\|_{p}^{r}+|\mu|\|h\|_{\infty} C_{r, q}^{r}\|v\|_{q}^{r} \\
& \leq d_{1}\left(\|u\|_{p}^{r}+\|v\|_{q}^{r}\right), \\
\int_{\Omega} f|u|^{\alpha}|v|^{\beta} d x & \\
& \leq\left\|f^{+}\right\|_{\infty} \int_{\Omega}\left(\frac{1}{\theta}|u|^{\alpha \theta}+\frac{1}{\theta^{\prime}}|v|^{\beta \theta^{\prime}}\right) d x \\
& \leq\left\|f^{+}\right\|_{\infty}\left(\frac{1}{\theta} S_{\alpha \theta, p}^{\alpha \theta}\|u\|_{p}^{\alpha \theta}+\frac{1}{\theta^{\prime}} S_{\beta \theta^{\prime}, q}^{\beta \theta^{\prime}}\|v\|_{q}^{\beta \theta^{\prime}}\right) \\
& \leq d_{2}\left(\|u\|_{p}^{s}+\|v\|_{q}^{s}\right),
\end{aligned}
$$

where $\theta=(\alpha+\beta) / \alpha, \theta^{\prime}=\theta /(\theta-1)=(\alpha+\beta) / \beta$, and

$$
\begin{aligned}
& d_{1}=\max \left\{|\lambda|\|g\|_{\infty} C_{r, p}^{r},|\mu|\|h\|_{\infty} C_{r, q}^{r}\right\}, \\
& d_{2}=\max \left\{\frac{\alpha}{\alpha+\beta}\left\|f^{+}\right\|_{\infty} S_{s, p}^{s}, \frac{\beta}{\alpha+\beta}\left\|f^{+}\right\|_{\infty} S_{s, q}^{s}\right\} .
\end{aligned}
$$

Thus $\left(\mathrm{H}_{3}\right)-\left(\mathrm{H}_{4}\right)$ are satisfied.

Case $2\left(1<\gamma<p \leq q<\alpha+\beta<p^{*}\right)$. Let $\alpha+\beta=r, \gamma=s$. Then $\left(\mathrm{H}_{1}\right)$ is satisfied.

For all $(u, v),\left(\phi_{1}, \phi_{2}\right) \in X$, we define potential operators $B, C: X \rightarrow X^{*}$ given by

$$
\begin{aligned}
\left\langle B(u, v),\left(\phi_{1}, \phi_{2}\right)\right\rangle= & \frac{\alpha}{\alpha+\beta} \int_{\Omega} f|u|^{\alpha-2} u|v|^{\beta} \phi_{1} d x \\
& +\frac{\beta}{\alpha+\beta} \int_{\Omega} f|u|^{\alpha}|v|^{\beta-2} v \phi_{2} d x, \\
\left\langle C(u, v),\left(\phi_{1}, \phi_{2}\right)\right\rangle= & \lambda \int_{\partial \Omega} g|u|^{s-2} u \phi_{1} d s \\
& +\mu \int_{\partial \Omega} h|v|^{s-2} v \phi_{2} d s .
\end{aligned}
$$

Similarly as before, we have

$$
r b(u, v)=\int_{\Omega} f|u|^{\alpha}|v|^{\beta} d x \leq d_{1}\left(\|u\|_{p}^{r}+\|v\|_{q}^{r}\right),
$$

$s c(u, v)=\lambda \int_{\partial \Omega} g|u|^{s} d s+\mu \int_{\partial \Omega} h|v|^{s} d s \leq d_{2}\left(\|u\|_{p}^{s}+\|v\|_{q}^{s}\right)$,

where

$$
\begin{aligned}
& d_{1}=\max \left\{\frac{\alpha}{\alpha+\beta}\left\|f^{+}\right\|_{\infty} S_{r, p}^{r}, \frac{\beta}{\alpha+\beta}\left\|f^{+}\right\|_{\infty} S_{r, q}^{r}\right\}, \\
& d_{2}=\max \left\{|\lambda|\|g\|_{\infty} C_{s, p}^{s},|\mu|\|h\|_{\infty} C_{s, q}^{s}\right\} .
\end{aligned}
$$

Thus $\left(\mathrm{H}_{2}\right)-\left(\mathrm{H}_{4}\right)$ are satisfied. 
By applying Theorem 10, we obtain the following.

Theorem 12. If (i) $2<\alpha+\beta<p \leq q<\gamma<p_{*}$ or (ii) $1<\gamma<p \leq q<\alpha+\beta<p^{*}$ is satisfied, then there exists $C_{0}>0$ such that, for $|\lambda|+|\mu|<C_{0}$, system (93) has at least two nonnegative solutions $\left(u_{0}^{+}, v_{0}^{+}\right)$in $\mathcal{N}^{+}$and $\left(u_{0}^{-}, v_{0}^{-}\right)$in $\mathcal{N}^{-}$ such that $u_{0}^{ \pm} \not \equiv 0, v_{0}^{ \pm} \not \equiv 0$.

Proof. Notice that (25)-(26) and (51)-(52) are satisfied provided $C_{0}$ is sufficiently small; we obtain all assumptions of Theorem 10 which are satisfied. Thus, by Theorem 10, there exist at least two solutions $\left(u_{0}^{+}, v_{0}^{+}\right) \in \mathcal{N}^{+}$and $\left(u_{0}^{-}, v_{0}^{-}\right) \in \mathcal{N}^{-}$. Moreover, since $J\left(u_{0}^{ \pm}, v_{0}^{ \pm}\right)=J\left(\left|u_{0}^{ \pm}\right|,\left|v_{0}^{ \pm}\right|\right)$and $\left(\left|u_{0}^{ \pm}\right|,\left|v_{0}^{ \pm}\right|\right) \in$ $\mathcal{N}^{ \pm}$, we may assume $u_{0}^{ \pm} \geq 0, v_{0}^{ \pm} \geq 0$. In the proof of Propositions 8 and 9 , we have proved that $c\left(u_{0}^{+}, v_{0}^{+}\right)>0$ and $b\left(u_{0}^{-}, v_{0}^{-}\right)>0$, respectively; thus $u_{0}^{+} \not \equiv 0, v_{0}^{+} \not \equiv 0, u_{0}^{-} \quad \equiv$ $0, v_{0}^{-} \not \equiv 0$.

(II) We consider the multiplicity of nonnegative solutions for the following $(p, q)$-Laplacian system with Dirichlet boundary conditions:

$$
\begin{aligned}
& -\Delta_{p} u+|u|^{p-2} u \\
& =\lambda f(x)|u|^{\gamma-2} u+\frac{\alpha}{\alpha+\beta} h(x)|u|^{\alpha-2} u|v|^{\beta}, \quad \text { in } \Omega, \\
& -\Delta_{q} v+|v|^{q-2} v \\
& =\mu g(x)|v|^{\gamma-2} v+\frac{\beta}{\alpha+\beta} h(x)|u|^{\alpha}|v|^{\beta-2} v, \quad \text { in } \Omega, \\
& u=v=0, \quad \text { on } \partial \Omega,
\end{aligned}
$$

where $\alpha, \beta, \gamma>1, \Omega \subset \mathbb{R}^{N}$ is a bounded domain with smooth boundary, $\lambda, \mu$ are parameters in $\mathbb{R} \backslash\{0\}$, and the weight functions $f, g, h \in C(\bar{\Omega})$ satisfy the following condition:

$\left(\mathrm{A}_{2}\right) f^{ \pm}=\max \{ \pm f, 0\} \quad \not \equiv 0, g^{ \pm}=\max \{ \pm g, 0\} \quad \not \equiv 0$, and $h^{+}=\max \{h, 0\} \not \equiv 0$.

We consider problem (103) in the framework of the Sobolev space $X=W_{0}^{1, p}(\Omega) \times W_{0}^{1, q}(\Omega)$.

A pair of functions $(u, v) \in X$ is said to be a weak solution of problem (103) if

$$
\begin{aligned}
& \int_{\Omega}\left(|\nabla u|^{p-2} \nabla u \nabla \phi_{1}+|u|^{p-2} u \phi_{1}\right) d x-\lambda \int_{\Omega} f|u|^{\gamma-2} u \phi_{1} d x \\
& -\frac{\alpha}{\alpha+\beta} \int_{\Omega} h|u|^{\alpha-2} u|v|^{\beta} \phi_{1} d x \\
& \int_{\Omega}\left(|\nabla v|^{q-2} \nabla v \nabla \phi_{2}+|v|^{q-2} v \phi_{2}\right) d x-\mu \int_{\Omega} g|v|^{\gamma-2} v \phi_{2} d x \\
& -\frac{\beta}{\alpha+\beta} \int_{\Omega} h|u|^{\alpha}|v|^{\beta-2} v \phi_{2} d x
\end{aligned}
$$

hold for all $\left(\phi_{1}, \phi_{2}\right) \in X$.
If $2<\alpha+\beta<p \leq q<\gamma<p^{*}$, let $\alpha+\beta=s, \gamma=r$; then, for all $(u, v),\left(\phi_{1}, \phi_{2}\right) \in X$, we define potential operators $B, C: X \rightarrow X^{*}$ given by

$$
\begin{aligned}
\left\langle B(u, v),\left(\phi_{1}, \phi_{2}\right)\right\rangle= & \lambda \int_{\Omega} f|u|^{r-2} u \phi_{1} d x \\
& +\mu \int_{\Omega} g|v|^{r-2} v \phi_{2} d x, \\
\left\langle C(u, v),\left(\phi_{1}, \phi_{2}\right)\right\rangle= & \frac{\alpha}{\alpha+\beta} \int_{\Omega} h|u|^{\alpha-2} u|v|^{\beta} \phi_{1} d x \\
& +\frac{\beta}{\alpha+\beta} \int_{\Omega} h|u|^{\alpha}|v|^{\beta-2} v \phi_{2} d x .
\end{aligned}
$$

Thus we have

$$
\begin{gathered}
r b(u, v)=\lambda \int_{\Omega} f|u|^{r} d x+\mu \int_{\Omega} g|v|^{r} d x, \\
s c(u, v)=\int_{\Omega} h|u|^{\alpha}|v|^{\beta} d x .
\end{gathered}
$$

It is clear that the corresponding energy functional of (103) is defined by

$$
J(u, v)=\frac{1}{p}\|u\|_{p}^{p}+\frac{1}{q}\|v\|_{q}^{q}-b(u, v)-c(u, v) .
$$

By standard Sobolev embedding theorems, we have

$$
\begin{gathered}
r b(u, v) \leq d_{1}\left(\|u\|_{p}^{r}+\|v\|_{q}^{r}\right), \\
s c(u, v)=\int_{\Omega} h|u|^{\alpha}|v|^{\beta} d x \leq d_{2}\left(\|u\|_{p}^{s}+\|v\|_{q}^{s}\right),
\end{gathered}
$$

where

$$
\begin{aligned}
& d_{1}=\max \left\{|\lambda|\|f\|_{\infty} S_{r, p}^{r},|\mu|\|g\|_{\infty} S_{r, q}^{r}\right\}, \\
& d_{2}=\max \left\{\frac{\alpha}{\alpha+\beta}\left\|h^{+}\right\|_{\infty} S_{s, p}^{s}, \frac{\beta}{\alpha+\beta}\left\|h^{+}\right\|_{\infty} S_{s, q}^{s}\right\} .
\end{aligned}
$$

Thus $\left(\mathrm{H}_{1}\right)-\left(\mathrm{H}_{4}\right)$ are satisfied.

If $1<\gamma<p \leq q<\alpha+\beta<p^{*}$, let $\alpha+\beta=r, \gamma=s$, and define potential operators $B, C: X \rightarrow X^{*}$ given by

$$
\begin{aligned}
& \left\langle B(u, v),\left(\phi_{1}, \phi_{2}\right)\right\rangle \\
& =\frac{\alpha}{\alpha+\beta} \int_{\Omega} h|u|^{\alpha-2} u|v|^{\beta} \phi_{1} d x \\
& \quad+\frac{\beta}{\alpha+\beta} \int_{\Omega} h|u|^{\alpha}|v|^{\beta-2} v \phi_{2} d x, \\
& \left\langle C(u, v),\left(\phi_{1}, \phi_{2}\right)\right\rangle \\
& =\lambda \int_{\Omega} f|u|^{s-2} u \phi_{1} d x+\mu \int_{\Omega} g|v|^{s-2} v \phi_{2} d x .
\end{aligned}
$$

Similarly, we have

$$
\begin{gathered}
r b(u, v)=\int_{\Omega} h|u|^{\alpha}|v|^{\beta} d x \leq d_{1}\left(\|u\|_{p}^{r}+\|v\|_{q}^{r}\right), \\
s c(u, v)=\lambda \int_{\Omega} f|u|^{s} d x+\mu \int_{\Omega} g|v|^{s} d x \leq d_{2}\left(\|u\|_{p}^{s}+\|v\|_{q}^{s}\right),
\end{gathered}
$$


where

$$
\begin{aligned}
& d_{1}=\max \left\{\frac{\alpha}{\alpha+\beta}\left\|h^{+}\right\|_{\infty} S_{r, p}^{r}, \frac{\beta}{\alpha+\beta}\left\|h^{+}\right\|_{\infty} S_{r, q}^{r}\right\}, \\
& d_{2}=\max \left\{|\lambda|\|f\|_{\infty} S_{s, p}^{s},|\mu|\|g\|_{\infty} S_{s, q}^{s}\right\} .
\end{aligned}
$$

Thus $\left(\mathrm{H}_{1}\right)-\left(\mathrm{H}_{4}\right)$ are satisfied.

Arguing as before, we obtain the following.

Theorem 13. If $2<\alpha+\beta<p \leq q<\gamma<p^{*}$ or $1<\gamma<p \leq$ $q<\alpha+\beta<p^{*}$, then there exists $C_{0}>0$ such that, for $|\lambda|+|\mu|<$ $C_{0}$, system (103) has at least two nonnegative solutions $\left(u_{0}^{+}, v_{0}^{+}\right)$ in $\mathscr{N}^{+}$and $\left(u_{0}^{-}, v_{0}^{-}\right)$in $\mathscr{N}^{-}$such that $u_{0}^{ \pm} \neq \equiv 0, v_{0}^{ \pm} \not \equiv 0$.

(III) Finally, we consider the multiplicity of nonnegative solutions for the following $(p, q)$-Laplacian system with boundary conditions containing both convex and concave nonlinearities:

$$
\begin{gathered}
-\Delta_{p} u+|u|^{p-2} u=0, \quad \text { in } \Omega, \\
-\Delta_{q} v+|v|^{q-2} v=0, \quad \text { in } \Omega, \\
\frac{\partial u}{\partial n}=\lambda f(x)|u|^{\gamma-2} u+\frac{\alpha}{\alpha+\beta} h(x)|u|^{\alpha-2} u|v|^{\beta} \quad \text { on } \partial \Omega, \\
\frac{\partial v}{\partial n}=\mu g(x)|v|^{\gamma-2} v+\frac{\beta}{\alpha+\beta} h(x)|u|^{\alpha}|v|^{\beta-2} v, \quad \text { on } \partial \Omega,
\end{gathered}
$$

where $\alpha, \beta, \gamma>1, \Omega \subset \mathbb{R}^{N}$ is a bounded domain with smooth boundary, $\lambda, \mu$ are parameters in $\mathbb{R} \backslash\{0\}$, and the weight functions $f, g, h \in C(\partial \Omega)$ satisfy the following condition:

$\left(\mathrm{A}_{3}\right) f^{ \pm}=\max \{ \pm f, 0\} \quad \not \equiv 0, g^{ \pm}=\max \{ \pm g, 0\} \quad \not \equiv 0$, and $h^{+}=\max \{h, 0\} \not \equiv 0$.

We consider problem (113) in the framework of the Sobolev space $X=W^{1, p}(\Omega) \times W^{1, q}(\Omega)$.

If $2<\alpha+\beta<p \leq q<\gamma<p_{*}$, then we let $\alpha+\beta=s, \gamma=r$, and define potential operators $B, C: X \rightarrow X^{*}$ given by

$$
\begin{aligned}
\left\langle B(u, v),\left(\phi_{1}, \phi_{2}\right)\right\rangle= & \lambda \int_{\partial \Omega} f|u|^{\gamma-2} u \phi_{1} d s \\
& +\mu \int_{\partial \Omega} g|v|^{\gamma-2} v \phi_{2} d s, \\
\left\langle C(u, v),\left(\phi_{1}, \phi_{2}\right)\right\rangle= & \frac{\alpha}{\alpha+\beta} \int_{\partial \Omega} h|u|^{\alpha-2} u|v|^{\beta} \phi_{1} d s \\
& +\frac{\beta}{\alpha+\beta} \int_{\partial \Omega} h|u|^{\alpha}|v|^{\beta-2} v \phi_{2} d s,
\end{aligned}
$$

for all $(u, v),\left(\phi_{1}, \phi_{2}\right) \in X$. By standard Sobolev embedding theorems, we have

$$
\begin{aligned}
& r b(u, v) \\
& =\lambda \int_{\partial \Omega} f|u|^{r} d s+\mu \int_{\partial \Omega} g|v|^{r} d s \leq d_{1}\left(\|u\|_{p}^{r}+\|v\|_{q}^{r}\right), \\
& s c(u, v)=\int_{\partial \Omega} h|u|^{\alpha}|v|^{\beta} d s \leq d_{2}\left(\|u\|_{p}^{s}+\|v\|_{q}^{s}\right),
\end{aligned}
$$

where

$$
\begin{aligned}
& d_{1}=\max \left\{|\lambda|\|f\|_{\infty} C_{r, p}^{r},|\mu|\|g\|_{\infty} C_{r, q}^{r}\right\}, \\
& d_{2}=\max \left\{\frac{\alpha}{\alpha+\beta}\left\|h^{+}\right\|_{\infty} C_{s, p}^{s}, \frac{\beta}{\alpha+\beta}\left\|h^{+}\right\|_{\infty} C_{s, q}^{s}\right\} .
\end{aligned}
$$

Thus $\left(\mathrm{H}_{1}\right)-\left(\mathrm{H}_{4}\right)$ are satisfied.

If $1<\gamma<p \leq q<\alpha+\beta<p_{*}$, then we let $\alpha+\beta=r, \gamma=s$, and define potential operators $B, C: X \rightarrow X^{*}$ given by

$$
\begin{aligned}
\left\langle B(u, v),\left(\phi_{1}, \phi_{2}\right)\right\rangle= & \frac{\alpha}{\alpha+\beta} \int_{\partial \Omega} h|u|^{\alpha-2} u|v|^{\beta} \phi_{1} d s \\
& +\frac{\beta}{\alpha+\beta} \int_{\partial \Omega} h|u|^{\alpha}|v|^{\beta-2} v \phi_{2} d s, \\
\left\langle C(u, v),\left(\phi_{1}, \phi_{2}\right)\right\rangle= & \lambda \int_{\partial \Omega} f|u|^{\gamma-2} u \phi_{1} d s \\
& +\mu \int_{\partial \Omega} g|v|^{\gamma-2} v \phi_{2} d s,
\end{aligned}
$$

for all $(u, v),\left(\phi_{1}, \phi_{2}\right) \in X$. By standard Sobolev embedding theorems, we have

$$
\begin{gathered}
r b(u, v)=\int_{\partial \Omega} h|u|^{\alpha}|v|^{\beta} d s \leq d_{1}\left(\|u\|_{p}^{r}+\|v\|_{q}^{r}\right), \\
s c(u, v) \lambda \int_{\partial \Omega} f|u|^{s} d s+\mu \int_{\partial \Omega} g|v|^{s} d s \\
\leq d_{2}\left(\|u\|_{p}^{s}+\|v\|_{q}^{s}\right),
\end{gathered}
$$

where

$$
\begin{aligned}
& d_{1}=\max \left\{\frac{\alpha}{\alpha+\beta}\left\|h^{+}\right\|_{\infty} C_{r, p}^{r}, \frac{\beta}{\alpha+\beta}\left\|h^{+}\right\|_{\infty} C_{r, q}^{r}\right\}, \\
& d_{2}=\max \left\{|\lambda|\|f\|_{\infty} C_{s, p}^{s},|\mu|\|g\|_{\infty} C_{s, q}^{s}\right\} .
\end{aligned}
$$

Thus $\left(\mathrm{H}_{1}\right)-\left(\mathrm{H}_{4}\right)$ are satisfied.

Arguing as before, we obtain the following.

If $2<\alpha+\beta<p \leq q<\gamma<p_{*}$ or $1<\gamma<p \leq q<\alpha+\beta<$ $p_{*}$, then there exists $C_{0}>0$ such that, for $|\lambda|+|\mu|<C_{0}$, system (113) has at least two nonnegative solutions $\left(u_{0}^{+}, v_{0}^{+}\right)$in $\mathcal{N}^{+}$and $\left(u_{0}^{-}, v_{0}^{-}\right)$in $\mathcal{N}^{-}$such that $u_{0}^{ \pm} \neq \equiv 0, v_{0}^{ \pm} \neq \equiv 0$.

\section{Conflict of Interests}

The author declares that there is no conflict of interests regarding the publication of this paper.

\section{Acknowledgment}

This work is supported by the Fundamental Research Funds for the Central Universities (2013B09914).

\section{References}

[1] A. Aghajani and J. Shamshiri, "Multiplicity of positive solutions for quasilinear elliptic p-Laplacian systems," Electronic Journal of Differential Equations, vol. 2012, no. 111, pp. 1-16, 2012. 
[2] Y. Bozhkov and E. Mitidieri, "Existence of multiple solutions for quasilinear systems via fibering method," Journal of Differential Equations, vol. 190, no. 1, pp. 239-267, 2003.

[3] G. A. Afrouzi and S. H. Rasouli, "A remark on the existence of multiple solutions to a multiparameter nonlinear elliptic system," Nonlinear Analysis: Theory, Methods \& Applications, vol. 71, no. 1-2, pp. 445-455, 2009.

[4] K. J. Brown and T.-F. Wu, "A fibering map approach to a potential operator equation and its applications," Differential and Integral Equations, vol. 22, no. 11-12, pp. 1097-1114, 2009.

[5] K. J. Brown and T.-F. Wu, "A semilinear elliptic system involving nonlinear boundary condition and sign-changing weight function," Journal of Mathematical Analysis and Applications, vol. 337, no. 2, pp. 1326-1336, 2008.

[6] H. Li, X.-P. Wu, and C.-L. Tang, "Multiple positive solutions for a class of semilinear elliptic systems with nonlinear boundary condition," Journal of Applied Mathematics and Computing, vol. 38, no. 1-2, pp. 617-630, 2012.

[7] M. Ishiwata, "Effect of topology on the multiplicity of solutions for some semilinear elliptic systems with critical Sobolev exponent," Nonlinear Differential Equations and Applications, vol. 16, no. 3, pp. 283-296, 2009.

[8] P. Drábek and S. I. Pohozaev, "Positive solutions for the $p$ Laplacian: application of the fibering method," Proceedings of the Royal Society of Edinburgh Section A, vol. 127, no. 4, pp. 703726, 1997. 


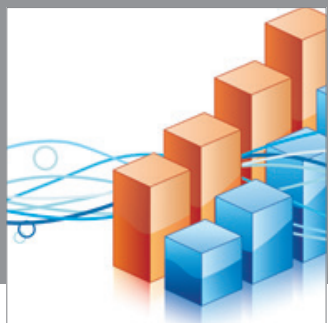

Advances in

Operations Research

mansans

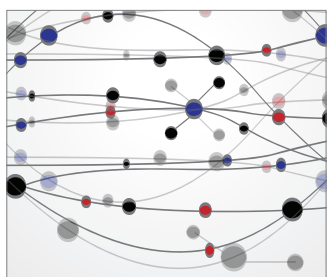

The Scientific World Journal
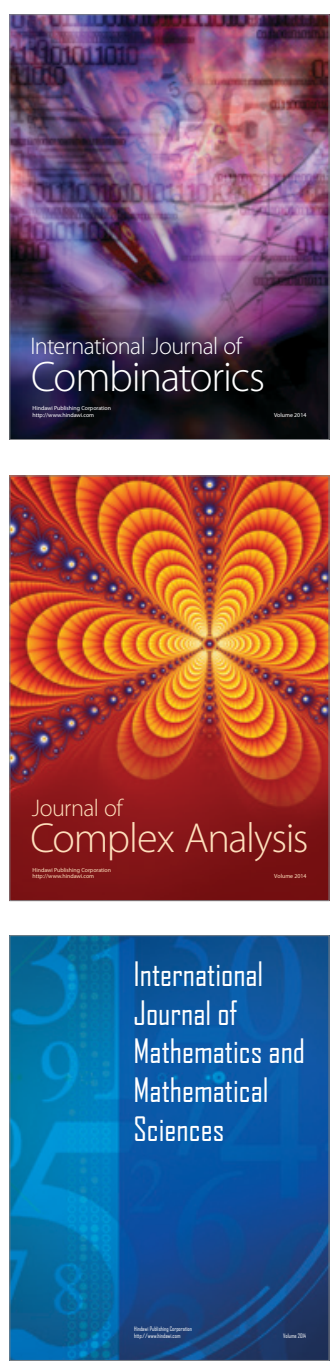
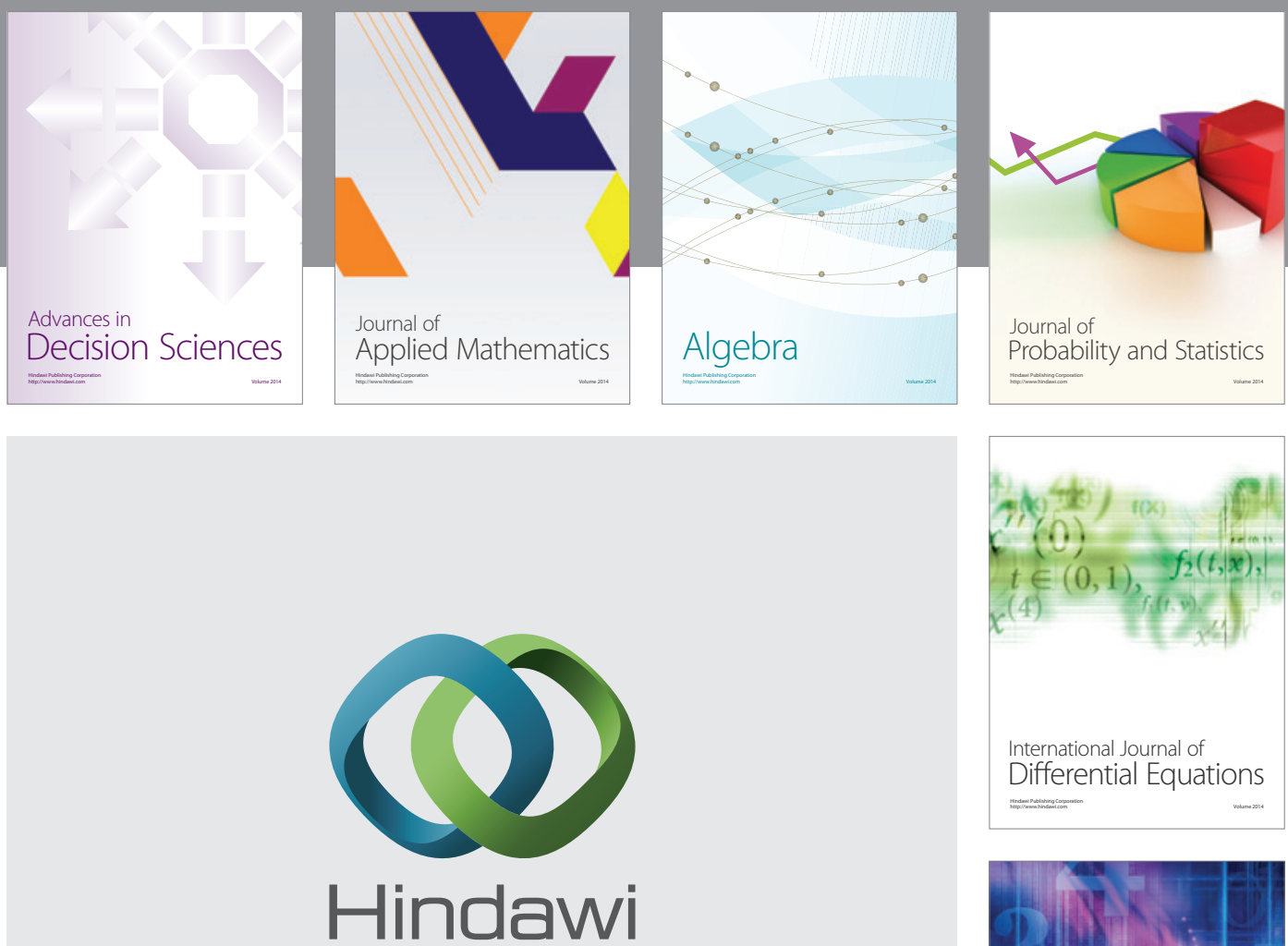

Submit your manuscripts at http://www.hindawi.com
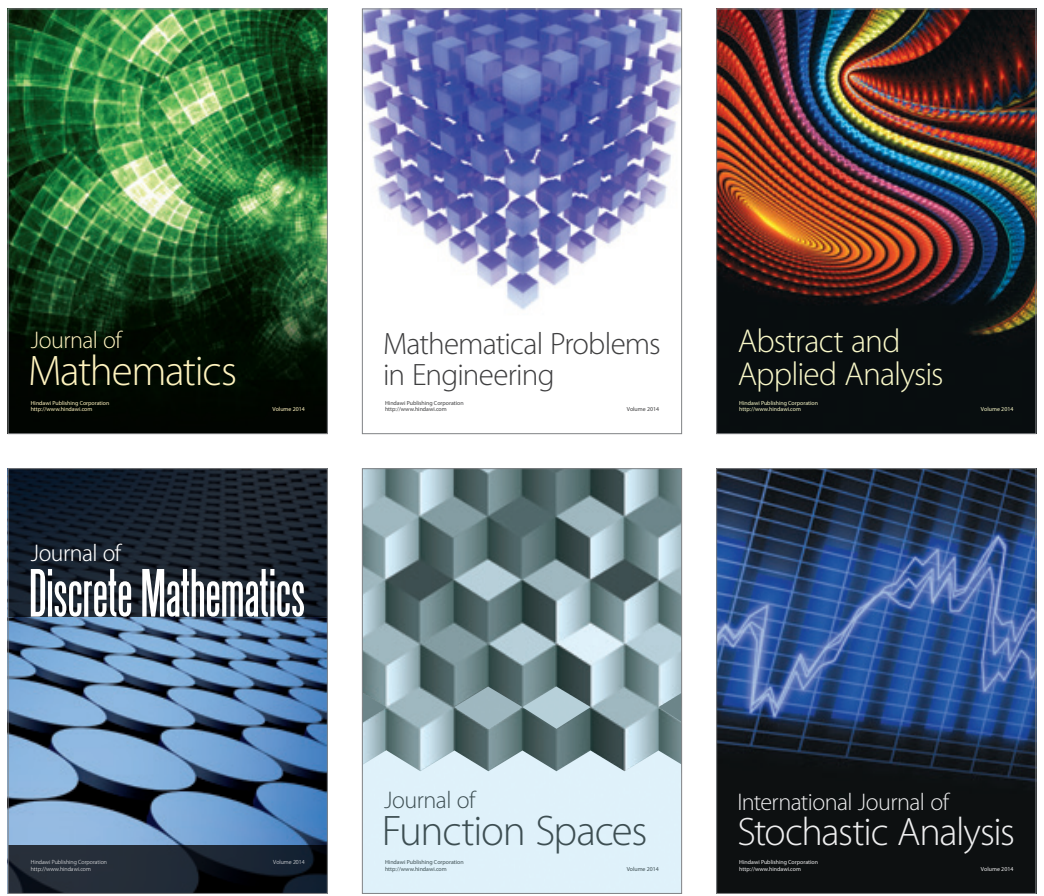

Journal of

Function Spaces

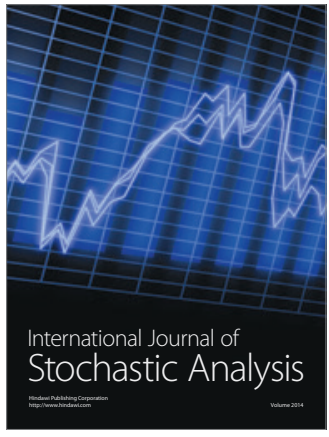

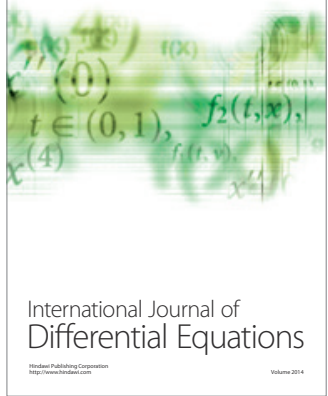
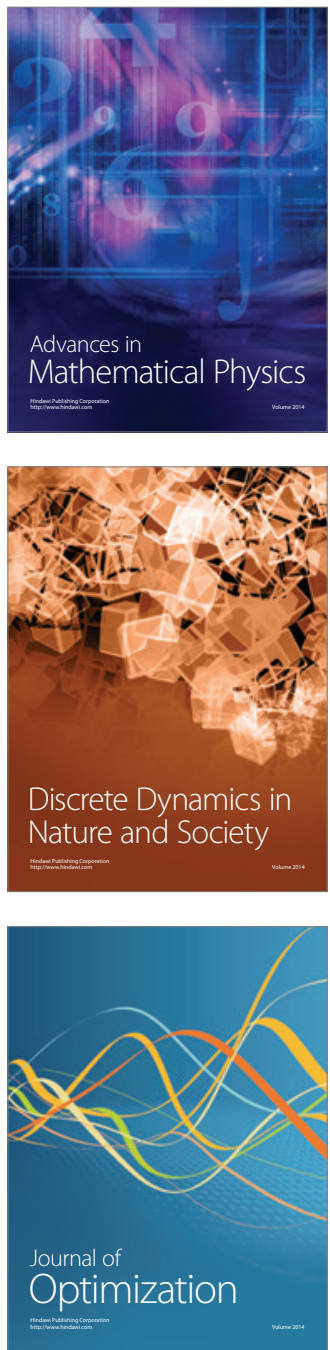